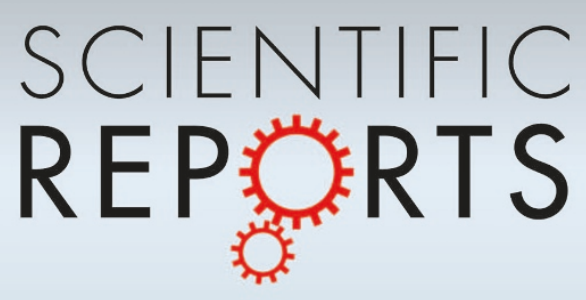

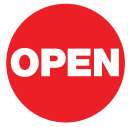

SUBJECT AREAS: APPLIED PHYSICS

CONDENSED MATTER PHYSICS

ELECTRONIC MATERIALS AND

DEVICES

MATERIALS PHYSICS

Received

5 July 2012

Accepted

25 July 2012

Published

8 August 2012

Correspondence and requests for materials should be addressed to

N.A.P. (n.a.porter@ leeds.ac.uk) or C.H.M. (c.h.marrows@leeds. ac.uk)

\section{Linear magnetoresistance in n-type silicon due to doping density fluctuations}

\author{
Nicholas A. Porter \& Christopher H. Marrows
}

School of Physics \& Astronomy, University of Leeds, Leeds LS2 9JT, United Kingdom.

We report the observation of a large linear magnetoresistance in the ohmic regime in commonplace commercial $n$-type silicon wafer with a $P$ dopant density of $(1.4 \pm 0.1) \times 10^{15} \mathrm{~cm}^{-3}$, and report measurements of it in the temperature range 30-200 K. It arises from the deformation of current paths, which causes a part of the Hall field to be detected at the voltage probes. In short, wide samples we found linear magnetoresistance as large as $4707 \%$ in an 8 tesla field at $35 \mathrm{~K}$. Sample geometry effects like these are commonplace in commercial Hall sensors. However, we found that the effect persisted in long, thin samples where the macroscopic current flow should be uniform between the voltage probes: we observed a magnetoresistance of $445 \%$ under the same conditions as above. We interpret this result as arising due to spatial fluctuations in the donor density, in the spirit of the Herring model.

W hilst a free electron gas has no magnetoresistance $(\mathrm{MR})^{1}$, the conventional theory of MR in metals and semiconductors relies upon a distribution of scattering times amongst the conducting carriers that cannot then be compensated by a unique Hall field ${ }^{2}$. For a material with a closed free electron Fermi surface (FS) and a principal charge carrier this leads to positive MR that is quadratic in weak magnetic fields, $B$, and saturates in strong magnetic fields according to:

$$
\frac{\Delta \rho}{\rho}=\frac{\rho(B)-\rho(0)}{\rho(0)}=\frac{A B^{2}}{1+C B^{2}},
$$

where $\rho$ is the resistivity. In the denominator of equation 1 it can be shown ${ }^{3}$ that $C B^{2}=\left(l_{\mathrm{MF}} / r_{\mathrm{c}}\right)^{2}=\left(\omega_{\mathrm{c}} \tau\right)^{2}$, where $\omega_{\mathrm{c}}$ is the cyclotron frequency, $\tau$ the mean free time between scattering events, $l_{\text {MFP }}$ the mean free path and $r_{\mathrm{c}}$ the cyclotron radius. In the strong field limit $\left(\omega_{\mathcal{c}} \tau \gg 1\right)$, electrons complete several orbits before scattering. In the weak field limit $\left(\omega_{\mathrm{c}} \tau \ll 1\right)$, only a fraction of these orbits are completed, and so $\Delta \rho / \rho \sim A B^{2}$, depending predominantly upon the anisotropy in relaxation times and cyclotron masses of the carriers ${ }^{3}$. Other galvanomagnetic effects are present in magnetically ordered materials ${ }^{4}$.

In the past decade some materials have emerged that break these previously established trends. Narrow gap semiconductors such as iron diantinomide ${ }^{5}$, the silver chalcogenides ${ }^{6,7}$, indium antimonide ${ }^{8}$, and nitrogen-doped indium arsenide ${ }^{9}$ have shown unexpectedly high MR over large temperature ranges. In each case, the MR was non-saturating, approximately linear at high fields, and was enhanced by strong disorder arising from impurity substitution $^{10,11}$ and spatial inhomogeneity of the sample stoichiometry ${ }^{6,9,12}$. In the last few years numerical modelling of large scale inhomogeneous inclusions using classical models has been used to describe this linear $\mathrm{MR}^{13,14}$. Other examples of systems displaying linear MR include MnAs-GaAs composites ${ }^{15}$, $\mathrm{PrFeAsO}^{16}$, epitaxial graphene ${ }^{17}$, and nanosheets of the topological insulator $\mathrm{Bi}_{2} \mathrm{Te}_{3}{ }^{18}$. Whilst all this recent interest has concerned a wide array of exotic materials, there are rather few examples of measurements in commonplace, commercialised semiconductor materials, of which $\mathrm{Si}$ is the prime example. Quasi-linear MR has been observed in $\mathrm{Si}^{19,20}$, but only measured at a single temperature, $77 \mathrm{~K}$. There have also been recent reports of large MR in high electric fields in intrinsic silicon $(\mathrm{i}-\mathrm{Si})^{21-23}$.

Here we have measured the MR in $n$-type commercial $\mathrm{Si}$ wafer in the ohmic regime over a wider temperature range, and find linear MR which we attribute to distortions in the paths of current flow at macroscopic and microscopic scales, leading to components of the Hall fields being measured at longitudinal voltage contacts. This phenomenon is known as "current jetting" and is described in detail by Pippard in Ref. 2. In samples where the width approaches the length, the macroscopic deformation of the current flow leads to just such an apparent magnetoresistance, as is used in some commercial Hall sensors ${ }^{24}$. This phenomenon can be reproduced within the numerical approach of Parish and Littlewood ${ }^{14}$. However the effect persists, albeit of smaller magnitude, in long narrow samples where macroscopic current jetting should have been eliminated. We argue that our data can be 
understood in terms of a subtle (but related) mechanism due to the weak disorder from spatially fluctuating conductivity that arises statistically from the random donor distribution ${ }^{25}$.

\section{Results}

Sample details. Si:P wafers with $3.6 \pm 0.3 \Omega \mathrm{cm}$ room temperature resistivity and dopant density $(1.4 \pm 0.1) \times 10^{15} \mathrm{~cm}^{-3}$ were used. This is just within the low doping regime where the MR is large $\mathrm{e}^{26}$. The doping density dependence of MR in $p$-type Si:B was addressed by Schoonus et al. ${ }^{22}$.

The net carrier density, $\langle n\rangle$, and an absolute value of the carrier mobility, $\mu$, were obtained as a function of temperature, $T$, using the van der Pauw method on square pieces of wafer (see supplementary Fig. S1 online). The MR was measured using four in-line contacts on samples cut from wafer with a thickness $t=530 \pm 50 \mu \mathrm{m}$. Indium ohmic contacts provided a four wire current-voltage $I-V$ characteristic that was ohmic at low bias throughout all temperatures. Measurements were performed in three orientations of field with respect to current density $\underline{j}$ : transverse $(\underline{j} \perp \underline{B})$ with the magnetic field out of the sample plane; perpendicular $(\underline{j} \perp \underline{B})$ with the magnetic field in plane; and longitudinal $(\underline{j} \| \underline{B})$.

MR dependence on sample geometry. A non-saturating linear MR in $\mathrm{Si}: \mathrm{P}$ can be generated by a classically understood method: altering the aspect ratio of the wafers, which causes deformation of current flow on a macroscopic scale, and can be used to enhance the response of devices in magnetic fields ${ }^{24}$. Solin et al. have shown that the geometrical enhancement in high mobility semiconductors in certain geometries such as Corbino disks can be used for practical applications such as read heads for high-density recording ${ }^{27,28}$. Geometrical enhancement of magnetoresistance can also be achieved in combination with high density current injection to generate regions of opposite doping ${ }^{29}$. When attempting to discern the physical MR of semiconductors however, as we shall do below, such geometrical effects are to be avoided.

Hence, in order to investigate geometrical effects in our material, we prepared nine samples with increasing width, $w$, with indium contacts positioned at a separation $l \sim 10 \mathrm{~mm}$ between current leads and $s \sim 3 \mathrm{~mm}$ between voltage probes. Photographs of these samples, labelled A-H, are shown in Fig. 1 with the dimensions, measured using vernier calipers, given in the caption. Perpendicular and transverse $\mathrm{MR}$ was measured at $35 \mathrm{~K}$ in the ohmic regime on all the samples. The results are shown in Fig. $1 \mathrm{a}$ and Fig. $1 \mathrm{~b}$ respectively.
In the perpendicular orientation, where the sample projection in the field direction does not change, the variation in MR between the samples is very small (Fig. 1a). In the transverse field shown in Fig. $1 \mathrm{~b}$ the projection of the sample in the magnetic field direction varied significantly. In this orientation, increasing the width of the samples dramatically enhanced the MR of the films. The widest sample, $H$, had a maximum $\Delta \rho / \rho=4707 \%$ at $8 \mathrm{~T}$. Sample $\mathrm{A}$, the narrowest sample, reached a maximum $\Delta \rho / \rho=445 \%$. Whilst this is over an order of magnitude smaller than in sample $H$, it is still a remarkably large value.

Geometrical transverse MR has been calculated for rectangular plates of homogeneous conductors with electrodes spanning the sample ends ${ }^{24,30}$ : our geometry differs slightly in that we have point-like contacts, but the same general conclusions hold. For the narrowest sample $A$, the net current flow would be approximately homogeneous providing a good indication of the physical MR intrinsic to the material. We now need to seek an explanation of this large intrinsic MR.

Intrinsic MR in Si. A further sample was measured with the following dimensions: width, $w=1.8 \pm 0.1 \mathrm{~mm}$; length, $l=5.5 \pm 0.5 \mathrm{~mm}$; and voltage probe separation, $s=2.0 \pm 0.4 \mathrm{~mm}$. The transverse MR in the ohmic regime, shown in Fig. 2a, was approximately linear up to high magnetic fields and showed no indication of saturation in $8 \mathrm{~T}$ fields at temperatures from $30-200 \mathrm{~K}$, and is much larger than at room temperature where the system barely enters the high-field regime ${ }^{26}$. The high field gradient of this plot is known as the Kohler slope (KS). The transverse data were capable of being reduced to a Kohler plot of $\Delta \rho / \rho$ versus $\omega_{\mathrm{c}} \tau$, shown in Fig. 2b. In weak and strong fields the MR scaled onto a unique curve according Kohler's law; $\Delta \rho / \rho=f\left(\omega_{\mathrm{c}} \tau\right)$, where $f$ is a scaling function, using the relationship $\omega_{\mathrm{c}} \tau=\mu B$. (Longitudinal measurements did not obey the Kohler rule but possessed a variable KS as a function of temperature as shown in supplementary Fig. S2 and Fig. S3 online for completeness.) In weak fields (shown in the inset of figure $2 \mathrm{~b}$ ) the departure from quadratic behaviour (shown as dashed line) occurred for less than half a completed cyclotron orbit, while linear MR was satisfied in strong fields for $\omega_{\mathrm{c}} \tau \gtrsim 2$.

We now discuss the possible sources of this linear MR. This phenomenon can arise in polycrystalline material with an open FS resulting from the averaging of saturating MR from closed orbits and nonsaturating MR from open orbits ${ }^{31}$. Such effects can also be caused by quantum linear magnetoresistance $(\mathrm{QLMR})^{8,32}$. The former can be disregarded for the single crystals used here and the closed silicon

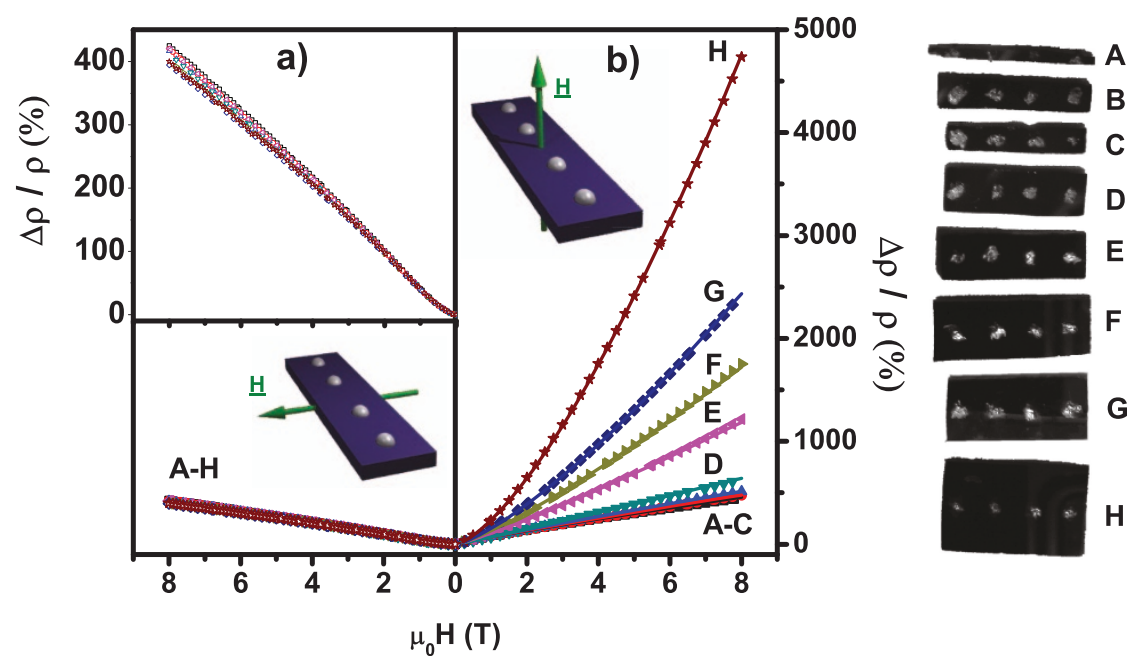

Figure $1 \mid$ Geometrical MR, measured in perpendicular (a) and transverse (b) field orientations at $35 \mathrm{~K}$ shown mirrored to one another using the right hand axis to encourage comparison. Wafers $\mathrm{A}-\mathrm{H}$ are shown with respective values of $\alpha=l / w: 10 \pm 1(\mathrm{~A}, \boldsymbol{\square}), 4.5 \pm 0.6(\mathrm{~B}, \boldsymbol{\bullet}), 3.7 \pm 0.5(\mathrm{C}, \mathbf{\Lambda}), 2.6 \pm 0.3$

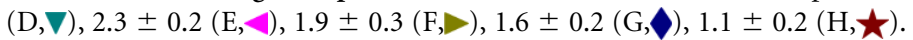




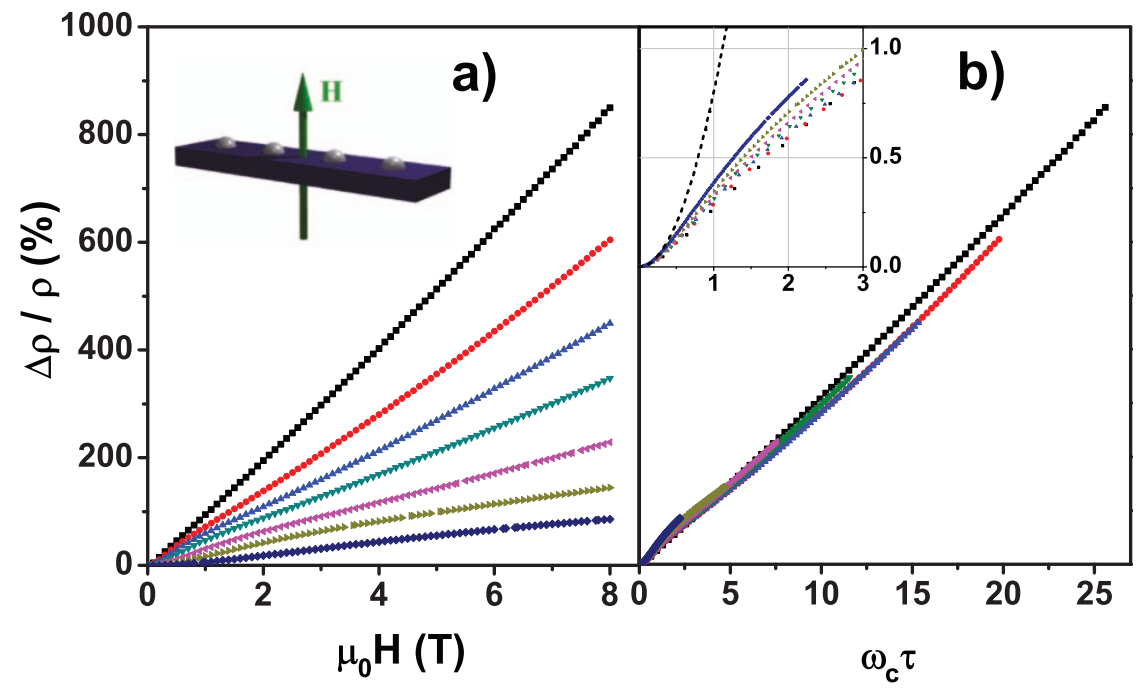

Figure $2 \mid$ Transverse MR at: $30(\square), 40(\bullet), 50(\Delta), 60(\nabla), 80(\triangleleft), 120(\downarrow)$ and $200 \mathrm{~K}(\triangleleft)$ in transverse magnetic fields for a long narrow sample. a) MR increased monotonically with decreasing temperature. In the strong magnetic field condition MR was non-saturating and approximately linear. b) MR at all temperatures reduced down to one unique curve provided $\mu B=\omega_{c} \tau$ conforming to Kohler's rule. The data is magnified in the inset to demonstrate the weak to strong field crossover at $\omega_{c} \tau \sim 1$ and universal scaling of the weak field MR. The strong field regime has fully set in for $\omega_{c} \tau \gtrless 2$. A quadratic law is plotted at weak fields, $\omega_{c} \tau \ll 1$ (dashed line).

conduction band energy surfaces. The latter requires a very small $n$ and effective mass, $m^{*}$ which generally limits it to metals and semimetals. As $m^{*} \sim 0.4 m_{e}$ in Si: $\mathrm{P}^{1}$, a field of $10 \mathrm{~T}$ would not produce QLMR above $30 \mathrm{~K}^{32}$ since the thermal energy would exceed the separation of the Landau levels, ruling out this mechanism here.

Aside from these possible causes, Pippard proposed four distinct mechanisms that could lead to strong field, non saturating linear MR in crystals ${ }^{2}$ : small angle scattering, magnetic breakdown, voids, and inhomogeneities. We now discuss each of these in turn.

The influence of small angle scattering is limited at higher temperatures, where electrons are scattered through larger angles. In Si:P at $100 \mathrm{~K}$, where a large positive MR is observed, the most effective phonons would scatter at $\sim 30^{\circ}$ suggesting that small angle scattering is not applicable ${ }^{2}$. Equally, magnetic breakdown from tunnelling between orbits in momentum-space is unlikely as the FS of the conduction band in Si lies far from the Brillouin zone boundary.

MR arising from spherical voids with a volume fraction $F$ scales as $0.486 F \omega_{\mathcal{c}} \tau$ in a transverse field and $0.637 F \omega_{\mathrm{c}} \tau$ in a longitudinal field ${ }^{2}$. As the magnitude of the transverse MR shown in Fig. 2a is much greater than the longitudinal MR (see supplementary Fig. S2 online) the observed MR does not agree with this hypothesis. In Fig. 2b, with the aspect ratio $l / w \sim 3$, the KS was $0.30 \pm 0.01$, implying a void volume fraction $F=0.62 \pm 0.02$ in our single crystal Si wafer, which is utterly implausible.

Thus, linear MR from inhomogeneities remains as the most probable cause of enhanced MR in strong fields. The limit of strong disorder has been used to explain the linear MR in the silver chalcogenides ${ }^{14}$. In this limit, models must resort to numerical techniques. However, silicon growth techniques aim to produce uniformly doped wafers and thus the disorder is likely to be comparatively weak. The suspicion that fluctuation in dopant density of pulled crystals may influence the conduction of semiconducting single crystals was the original motivation for Herring's perturbative mode ${ }^{25}$, although there was little direct experimental evidence available at that time. The spatial variations in Hall coefficient that these defects give rise to were predicted to prevent saturation of the transverse MR whilst having no effect on the saturating longitudinal MR. For instance, in the non-degenerate weakly doped sample discussed here, the average impurity separation is $\sim 90 \mathrm{~nm}$. The mean free path at $200 \mathrm{~K}$, $l_{\mathrm{MFP}}=(\mu / e)\left(3 m^{*} k_{\mathrm{B}} T\right)^{1 / 2}$, where $e$ is the electron charge and $k_{\mathrm{B}}$ is
Boltzmann's constant, would be $\sim 92 \mathrm{~nm}^{33}$. As the electrons will scatter on a lengthscale comparable to the impurity separation they can be susceptible to fluctuations in the concentration of ionised donors, $N_{\mathrm{i}}$, on lengthscales greater than their mean free path. Herring suggested that the fractional variance (FV) of the number of ionised donors $\left\langle\left(N_{\mathrm{i}}-\left\langle N_{\mathrm{i}}\right\rangle\right)^{2}\right\rangle /\left\langle N_{\mathrm{i}}\right\rangle^{2}$ was the relevant measure of the fluctuation magnitude. If this value was significant over lengthscales greater than a suitably defined effective mean free path, $l_{\text {eff }}$, and the Debye screening length, $\lambda_{\mathrm{D}}$, then the inhomogeneity that results from the distribution of random impurities would create uncompensated Hall fields that contribute significantly to a linear $\mathrm{MR}^{25}$.

The fractional variance should hence be limited on a lengthscale $\lambda_{D}=\sqrt{\epsilon k_{B} T /\left(\langle n\rangle e^{2}\right)}$, where $\epsilon$ is the Si:P permittivity, in lightly doped samples, and by $l_{\text {eff }}=\left(2 /\left(\omega_{\mathrm{c}} \tau\right)^{2}\right)^{1 / 3} l_{\mathrm{MFP}}$ in highly doped samples. Isotropic, saturating MR such as in equation 1 is assumed in the case of homogeneous doping. When donor density fluctuations are introduced, the effective MR for transverse isotropic fluctuations in $N_{\mathrm{i}}$ can be written as ${ }^{25}$ :

$$
\frac{\Delta \rho_{\text {eff }}}{\rho_{\text {eff }}} \sim \frac{\Delta \rho}{\rho}+\frac{B}{n e p(0)} \zeta \frac{\left\langle\left(N_{i}-\left\langle N_{i}\right\rangle\right)^{2}\right\rangle}{\left\langle N_{i}\right\rangle^{2}},
$$

where $\zeta$ has a numerical value that depends upon the conductivity anisotropy. In the isotropic case relevant here, $\zeta=\pi / 4$.

To estimate the $\mathrm{FV}$ of a random distribution of impurities over the relevant lengthscales, we simulated a cubic crystal consisting of $10^{12}$ atoms, substituting random host atoms for ionised impurities. The $\mathrm{FV}$ of the impurities probed over different lengthscales is shown in Fig. 3a. The average density of ionised impurities at 50 and $200 \mathrm{~K}$ was taken from supplementary Fig. S1 assuming that $N_{\mathrm{i}} \approx n$ in ohmic conduction. Included in Fig. 3 a are the lower limits on the FV at each temperature given by the lengthscales $\lambda_{\mathrm{D}}$ and $l_{\mathrm{eff}}$, the latter calculated for $\omega_{\mathrm{c}} \tau=2$. (We use this value as representative of the point at which the strong field, linear MR regime has fully set in, on the basis of the data presented in figure 2.) These limitations suggest that the FV should be no more than 0.27 at $50 \mathrm{~K}$ and 0.77 at $200 \mathrm{~K}$.

To see if these values of the FV in impurity density are sufficient to produce the observed linear MR, fits to equation 2 were performed using the measured $\mu, \rho(0)$, and $\langle n\rangle$ values with only the FV and $A$ as fitting parameters. The fits are shown in Fig. $3 \mathrm{~b}$, showing that a FV of 0.34 is required at $50 \mathrm{~K}$ and falls only a little to 0.21 at $200 \mathrm{~K}$. The fact 


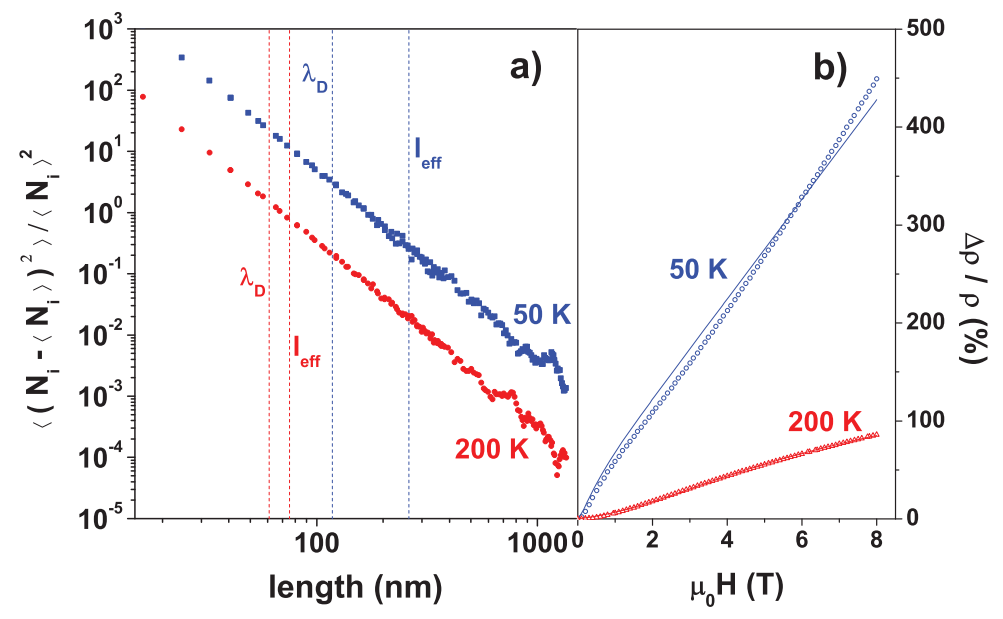

Figure 3 Interpretation of the observed MR within the Herring model. (a) Simulation of the impurity concentration fluctuations of a cubic crystal of $10^{12}$ atoms on different lengthscales. The vertical lines mark the Debye screening length, $\lambda_{\mathrm{D}}$, and effective mean free path, $l_{\text {eff }}$, at $50 \mathrm{~K}$ and $200 \mathrm{~K}$. These parameters provide an upper limit for the FV to which the electron will be sensitive. For this impurity concentration the FV is limited by $l_{\text {eff }}$ at both temperatures. b) Fits (lines) to the transverse MR (symbols) at 50 and $200 \mathrm{~K}$ according to the simplified model of Herring in equation 2 . The number of points plotted for the experimental data are reduced so as not to obscure the fit.

that the transverse MR obeys Kohler's rule indicates that the level of conductivity fluctuations must vary weakly with temperature, so it is to be expected that these values not to differ to greatly. Whilst not fulfilling the condition that $\mathrm{FV} \ll 1$ required for the perturbative approach to be strictly correct, these values are small enough $(\mathrm{FV}<1)$ that we can classify the level of disorder as being quite weak. (In any case, the numerical results of Parish and Littlewood, described in Ref. 13 and Ref. 14, are certainly suggestive of linear magnetoresistance away from the perturbative regime, indicating that the correct basic physics is captured by this approach.) At $50 \mathrm{~K}$, it is expected that the value for $\omega_{c} \tau$ for which these conditions are satisfied should be 2 ; from our fits, the value for $\omega_{c} \tau=2.25$ is in reasonable agreement. Overall, and in spite of the disorder levels not being so weak as to strictly justify its perturbative approach, it does seem that the classical Herring model provides a satisfactory physical picture for explaining for our observations.

\section{Discussion}

Hence, we conclude that the large non-saturating MR we observe here relies upon application of Hall fields to the voltage contacts, which arise either at the macroscopic or microscopic scale. Microscopically, the distribution of ionised donors is sufficiently disordered that current distortions arise from the local fluctuating conductivities. The donor distribution in this study is random, defined by a three-dimensional Poisson distribution, but with modern lithographic and ion implantation techniques, the local density could be tailored to maximise this effect. Alternatively, for a thin sample, lithography techniques could produce an array of top gates that could tune the local density of ionised donors to provide strong disorder by locally modifying the Hall constant, providing a MR response that can be tailored with an applied electric field.

If the carrier concentration is decreased, as is the case for very lightly doped silicon, the linear MR from the FV in equation 2 is limited by the Debye electron screening length. This limitation from screening could perhaps be overcome by the large electric fields resulting from the concentration of conduction electrons. As $\lambda_{\mathrm{D}} \propto\langle n\rangle^{-1 / 2}$, in the non-quasineutral Mott-Gurney regime, $\lambda_{\mathrm{D}}$ would be reduced without changing the $\mathrm{FV}$ of the ionised impurities. As the FV would remain large, with the reduced limitation from $\lambda_{\mathrm{D}}$, a large FV may produce a large MR even in intrinsic samples. This could help to explain the large MR in high electric fields reported for intrinsic silicon (i-Si $)^{21-23}$. For instance, with typically $\left\langle N_{\mathrm{i}}\right\rangle \sim 10^{12} \mathrm{~cm}^{3}$ at $300 \mathrm{~K},\left\langle\left(N_{\mathrm{i}}-\left\langle N_{\mathrm{i}}\right\rangle\right)^{2}\right\rangle /\left\langle N_{\mathrm{i}}\right\rangle^{2} \sim 0.01$. By increasing the source-drain current to inject more carriers such that $\left\langle n_{\text {inj }}\right\rangle \sim$ $10\langle n\rangle$ this value would increase to $\sim 0.5$. It is possible that the significant enhancement that could arise in both Mott-Gurney regime and during electrical breakdown could increase the MR to produce the large measured values ${ }^{21,22}$.

\section{Methods}

In all wafers the $\langle 001\rangle$ crystal vector was normal to the surface. High angle XRD verified that the wafers were cleaved along $\langle 110\rangle$ orientations such that the current, $\underline{j}$, was parallel to $\langle 110\rangle$ in all measurements.

Contacts were made to the wafer surface by mechanical cleavage through the native oxide with a diamond scribe followed by impression of indium. The contacts were then annealed at $350^{\circ} \mathrm{C}$ for 10 minutes. When the contacts were made, aluminium wire was attached using a wedge bonder from the sample holder to the indium. A $3 \times 3 \mathrm{~mm}^{2}$ square wafer was used for van der Pauw measurements using magnetic fields of $\pm 1.5 \mathrm{~T}$ to ascertain Hall voltages. All magnetoresistance measurements were performed in a gas flow cryostat using a four probe in-line DC technique at low bias in the ohmic regime, with the current bias adjusted to maintain roughly $5 \mathrm{mV}$ between the voltage probes.

1. Ashcroft, N. W. \& Mermin, N. D. Solid State Physics (Harcourt Brace, Fort Worth, 1976).

2. Pippard, A. B. Magnetoresistance in Metals (Cambridge University Press, Cambridge, 1989).

3. Sommerfeld, A. \& Frank, N. H. The statistical theory of thermoelectric, galvanoand thermomagnetic phenomena in metals. Rev. Mod. Phys. 3, 1-42 (1931).

4. O’Handley, R. C. Modern Magnetic Materials (Wiley, New York, 2000).

5. Petrovic, C. et al. Anisotropy and large magnetoresistance in the narrow-gap semiconductor $\mathrm{FeSb}_{2}$. Phys. Rev. B 67, 155205 (2003).

6. Xu, R. et al. Large magnetoresistance in non-magnetic silver chalcogenides. Nature 390, 57-60 (1997)

7. Husmann, A. et al. Megagauss sensors. Nature 417, 421 (2002).

8. Hu, J. \& Rosenbaum, T. F. Classical and quantum routes to linear magnetoresistance. Nature Mater. 7, 697-700 (2008).

9. Patanè, A. et al. Effect of low nitrogen concentrations on the electronic properties of InAs ${ }_{1-x} \mathrm{~N}_{x}$. Phys. Rev. B 80, 115207 (2009).

10. Hu, R. et al. Colossal positive magnetoresistance in a doped nearly magnetic semiconductor. Phys. Rev. B 77, 085212 (2008).

11. Manyala, N. et al. Magnetoresistance from quantum interference effects in ferromagnets. Nature 404, 581 (2000).

12. Hu, J., Rosenbaum, T. F. \& Betts, J. B. Current jets, disorder, and linear magnetoresistance in the silver chalcogenides. Phys. Rev. Lett. 95, 186603 (2005).

13. Parish, M. M. \& Littlewood, P. B. Non-saturating magnetoresistance in heavily disordered semiconductors. Nature 426, 162-165 (2003).

14. Parish, M. M. \& Littlewood, P. B. Classical magnetotransport of inhomogeneous conductors. Phys. Rev. B 72, 094417 (2005).

15. Johnson, H. G., Bennett, S. P., Barua, R., Lewis, L. H. \& Heiman, D. Universal properties of linear magnetoresistance in strongly disordered MnAs-GaAs composite semiconductors. Phys. Rev. B 82, 085202 (2010). 
16. Bhoi, D., Mandal, P., Choudhury, P., Pandya, S. \& Ganesan, V. Quantum magnetoresistance of the PrFeAsO oxypnictide. Appl. Phys. Lett. 98, 172105 (2011).

17. Friedman, A. L. et al. Quantum linear magnetoresistance in multilayer epitaxial graphene. Nano. Lett. 10, 3962 (2010).

18. Wang, X., Du, Y., Dou, S. \& Zhang, C. Room temperature giant and linear magnetoresistance in topological insulator $\mathrm{Bi}_{2} \mathrm{Te}_{3}$ nanosheets. Phys. Rev. Lett. 108, 266806 (2012).

19. Baranskii, P. I., Babich, V. M., Venger, E. F. \& Dotsenko, Yu. P. The features of magnetoresistance of $n$-Si doped with phosphorous from the melt and by nuclear transmutation. Semicond. Phys. Quantum Electron. Optoelectron. 3, 449-452 (2000).

20. Baranskyy, P. I., Gaydar, G. P. \& Litovchenko, P. Influence of the annealing of silicon crystals at $1200^{\circ} \mathrm{C}$ on the Hall effect and magnetoresistance. Semicond. Phys. Quantum Electron. Optoelectron. 5, 231-234 (2002).

21. Delmo, M. P., Yamamoto, S., Kasai, S., Ono, T. \& Kobayashi, K. Large positive magnetoresistive effect in silicon induced by the space-charge effect. Nature 457, 1112-1115 (2009)

22. Schoonus, J. J. H. M., Haazen, P. P. J., Swagten, H. J. M. \& Koopmans, B Unravelling the mechanism of large room-temperature magnetoresistance in silicon. J. Phys. D: Appl. Phys. 42, 185011 (2009).

23. Ciccarelli, C., Park, B. G., Ogawa, S., Ferguson, A. J. \& Wunderlich, J. Gate controlled magnetoresistance in a silicon metal-oxide-semiconductor field-effecttransistor. Appl. Phys. Lett. 97, 082106 (2010).

24. Popovic, R. S. Hall Effect Devices (Institute of Physics Publishing, London, 2004), 2nd edn.

25. Herring, C. Effect of random inhomogeneities on electrical and galvanomagnetic measurements. J. Appl. Phys. 31, 1939-1953 (1960).

26. Porter, N. A. \& Marrows, C. H. Dependence of magnetoresistance on dopant density in phosphorous doped silicon. J. Appl. Phys. 109, 07 C703 (2011).

27. Solin, S. A., Thio, T., Hines, D. R. \& Heremans, J. J. Enhanced room-temperature geometric magnetoresistance in inhomogeneous narrow-gap semiconductors. Science 289, 1530-1532 (2000).

28. Solin, S. A. et al. Nonmagnetic semiconductors as read-head sensors for ultrahigh-density magnetic recording. Appl. Phys. Lett. 80, 4012-4014 (2002).
29. Wan, C., Zhang, X., Gao, X., Wang, J. \& Tan, X. Geometrical enhancement of lowfield magnetoresistance in silicon. Nature 477, 304 (2011).

30. Heremans, J. Solid state magnetic field sensors and applications. J. Phys. D Appl Phys. 26, 1149 (1993).

31. Lifshitz, I. M., Azbel, M. I. \& Kaganov, M. I. On the theory of galvanomagnetic effects in metals. Sov. Phys. JETP 3, 143-145 (1956).

32. Abrikosov, A. A. Quantum linear magnetoresistance; solution of an old mystery. J. Phys. A: Math. Gen. 36, 9119 (2003).

33. Weber, L. \& Gmelin, E. Transport properties of silicon. Appl. Phys. A 53, 136-140 (1991).

\section{Acknowledgements}

This work was supported by the EPSRC. We acknowledge useful discussions with Prof. Bryan Gallagher.

\section{Author contributions}

N.A.P. fabricated and measured the samples, analysed the data, and performed the numerical calculations. C.H.M. supervised the project. Both authors wrote the manuscript.

\section{Additional information}

Supplementary information accompanies this paper at http://www.nature.com/ scientificreports

Competing financial interests: The authors declare no competing financial interests.

License: This work is licensed under a Creative Common

Attribution-NonCommercial-NoDerivative Works 3.0 Unported License. To view a copy of this license, visit http://creativecommons.org/licenses/by-nc-nd/3.0/

How to cite this article: Porter, N.A. \& Marrows, C.H. Linear magnetoresistance in $n$-type silicon due to doping density fluctuations. Sci. Rep. 2, 565; DOI:10.1038/srep00565 (2012) 